\title{
Метод определения функциональной живучести при децентрализованном управлении интеллектуальным сервисом
}

\author{
Н. А. Князева \\ Одесская национальная академия пищевых технологий, ул. Канатная, 112, Одесса, 65082, Украина \\ e-mail: knyazeva@ukr.net, http://orcid.org/0000-0002-1523-6775
}

В статье предложен метод определения функииональной живучести интеллектуальной надстройки, осущеествляющей децентрализованное управление процессом предоставления интеллектуальных сервисов, получены выражения для оиенки функииональной живучести системы предоставления интеллектуальных сервисов выполнения некоторого подмножества функиий, которые реализуют ичель функционирования системы (предоставления интеллектуальных сервисов определенного класса), а также выполнения хотя бы одной функиии из подмножества (предоставления хотя бы одного вида интеллектуального сервиса определенного класса). Полученные выражения целесообразно использовать при проектировании системы управления интеллектуальным сервисом, при выборе принципа управления, при решении задач повышения функциональной живучести системы управления интеллектуальным сервисом.

Ключевые слова: Функциональная живучесть; Интеллектуальная надстройка; Децентрализованное управление; Интеллектуальный сервис.

\section{Метод визначення функціональної живучості при децентралізованому управлінні інтелектуальним сервісом}

\section{Н. О. Князева}

Одеська національна академія харчових технологій, вул. Канатна, 112, Одеса, 65082, Україна

\begin{abstract}
У статті запропоновано метод визначення функиіональної живучості інтелектуальной надбудови, що здійснює децентралізоване управління проиесом надання інтелектуальних сервісів, отримані вирази для оиінки функціональної живучості системи надання інтелектуальних сервісів - виконання деякої підмножини функцій, які реалізують мету функціонування системи (надання інтелектуальних сервісів певного класу), а також виконання хоча б однієї функиії з підмножини (надання хоча б одного виду інтелектуального сервісу певного класу). Отримані вирази доцільно використовувати при проектуванні системи управління інтелектуальним сервісом, при виборі принципу управління, при вирішенні задач підвищення функціональної живучості системи управління інтелектуальним сервісом.
\end{abstract}

Ключові слова: Функціональна живучість; Інтелектуальна надбудова; Децентралізоване управління; Інтелектуальний сервіс.

\section{DOI: http://dx.doi.org/10.15673/ret.v53i2.598}

(C) The Author(s) 2017. This article is an open access publication

This work is licensed under the Creative Commons Attribution 4.0 International License (CC BY) http://creativecommons.org/licenses/by/4.0/

\section{1 Введение}

Интенсивное развитие телекоммуникационных сетей (ТКС) - переход к сетям следующего поколения NGN (Next Generation Network) и сетям будущего поколения FGN (Future Generation Network) - обусловливает особую важность вопросов оценки и обеспечения живучести ТКС. Под живучестью ТКС понимают способность сохранять и восстанавливать выполнение основных функций в заданном объеме и на протяжении заданного времени в случае изменения структуры ТКС и условий ее функционирования вследствие некоторых неблагоприятных внешних воздействий $[1,2]$. Вопросам теории живучести систем различного назначения посвящены работы В.М. Вишневского, Ю.Ю. Громова, А.Г. Додонова, Ю.И. Стекольникова, И.А. Рябинина и др. В работах данных авторов и в других работах в соответствии со сформулированными принципами относительно оптимизации характеристик живучести различных типов систем [2], руководство которыми приводит к положительному эффекту в деле обеспечения живучести систем, предложены методы оценки и обес- 
печения структурной и функциональной живучести в системах различного назначения, в том числе в ТКС [1-6].

В начале XXI столетия существенно возрос спрос на интеллектуальные сервисы, например, такие как бронирование билетов, предоставление банковской информации, получение информации о погоде в конкретной местности и т.д. В данный момент список интеллектуальных сервисов значительно вырос, появились такие сервисы как: Premium Rate Service (звонки с начислением дополнительной оплаты, например, за доступ к информационным ресурсам или за участие в телефонных лотереях, голосованиях и т.п.), Prepaid Calling (звонки по предоплате с доступом абонентов по паролям), Least Cost Routing (маршрутизация по наиболее выгодному маршруту), VAS (Value Added Services, услуги с добавленной стоимостью) и ряд других. Предоставляет подобные сервисы платформа, в основу которой входит интеллектуальная надстройка. Интеллектуальная надстройка представляет собой по существу систему управления предоставлением интеллектуальных сервисов.

Активное развитие интеллектуальных сервисов (ИС) и необходимость выполнения определенных требований к качеству ИС, определяет важность задач оценки и обеспечения функциональной живучести ин- теллектуальной настройки. В работе [5] предложен метод определения функциональной живучести интеллектуальной настройки, осуществляющей централизованное управление процессом предоставления ИС, получены выражения для оценки функциональной живучести системы предоставления ИС - выполнения некоторого подмножества функций $f^{*}$, которые реализуют цель функционирования системы (предоставления ИС определенного класса), а также выполнения хотя бы одной функции из подмножества $f^{*}$ (предоставления $i$-го вида ИС определенного класса).

Исходя из того, что существует тенденция роста количества абонентов и, соответственно, спроса на ИС, для повышения качества управления предоставлением ИС в NGN целесообразно использовать интеллектуальную надстройку 3 децентрализованным принципом управления (ИНДПУ). При использовании ИНДПУ вопросы определения функциональной живучести, рассмотренные в настоящей работе, являются безусловно актуальными.

\section{2 Основная часть}

На рисунке 1 представлена архитектура NGN с ИНДПУ [7].

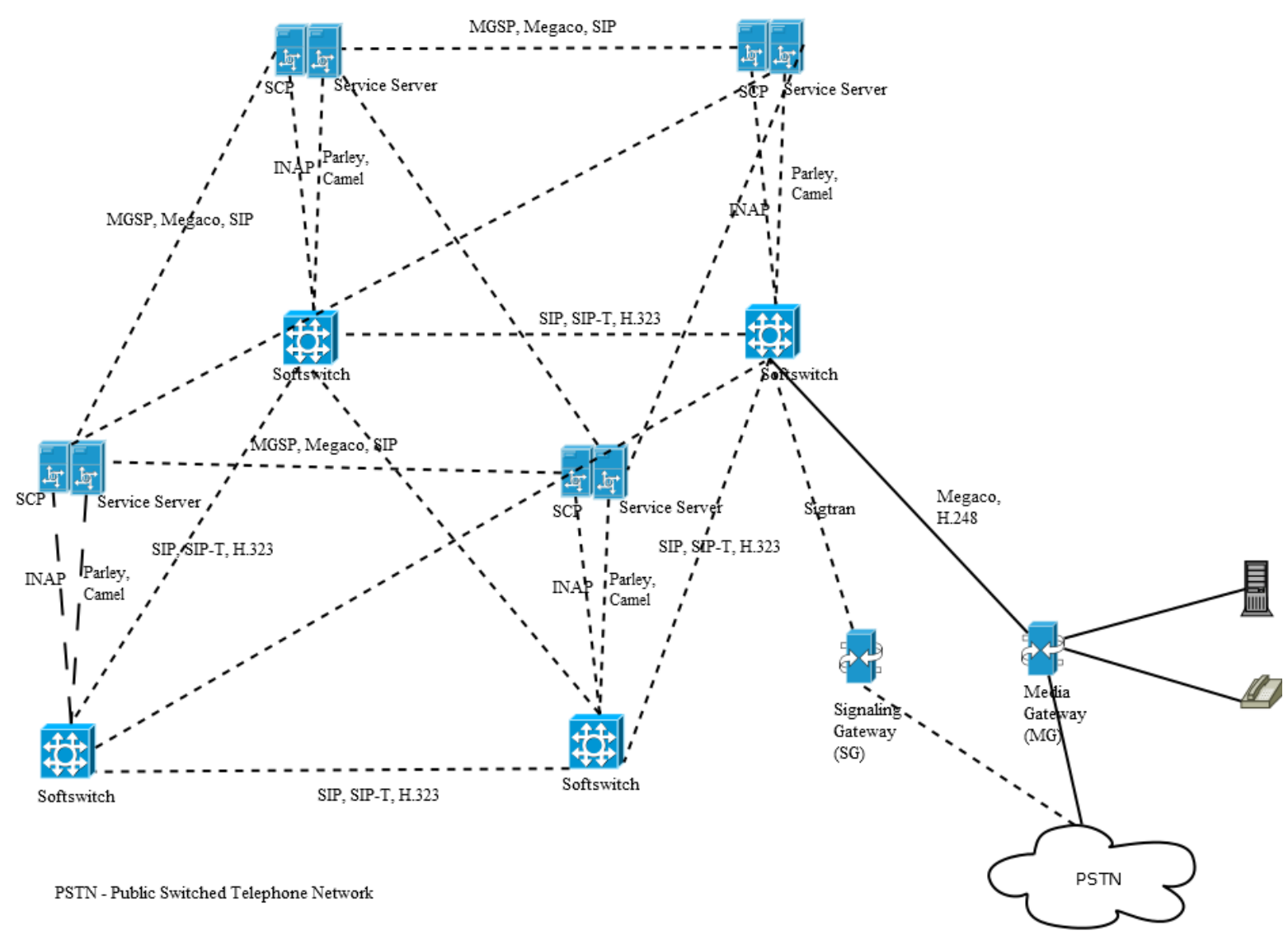

Рисунок 1 - Архитектура NGN с ИНДПУ

Как видно из рисунка 1, предусмотрено существование нескольких территориально разнесённых районов. В каждом 3 них установлен программный коммутатор Softswitch, который осуществляет управление районной транспортной сетью и исполняет функцию коммутации сервиса (на рисунке 1 такое подключение изображено лишь для одного Softswitch).

Реализовано четкое разделение NGN на сеть передачи данных (транспортную платформу) и интеллектуальную надстройку. Если в районной сети используется 
не только технология с коммутацией пакетов, но и с коммутацией каналов, то необходимо установить шлюз сигнализации SG (Signaling Gateway) в сети сигнализации и медиашлюз MG (Media Gateway) в сети передачи данных. Сервер сервисов исполняет логику сложных ИС. Реализация ИС осуществляется на базе узлов управления сервисами (SCP - Service Control Point). Серверы в интеллектуальной надстройке соединены по принципу «каждый с каждым». При обмене информацией между серверами могут использоваться протоколы MGCP, MEGACO, SIP, Н-323. Связь между Softswitch и сервером осуществляется через открытые API (Application Programming Interface - прикладной программный интерфейс) - Parlay, Camel.

При такой архитектуре заявка на ИС поступает от абонента к одному из пунктов сигнализации (SG). Softswitch посылает запрос к серверу, который непосредственно с ним связан, на исполнение заявки. Когда сервер данного района не может обслужить заявку, он обращается к серверу другого района, способного это осуществить. Для определения, к какому серверу следует обращаться, целесообразно использовать матрицу вероятностей передачи заявки с текущего сервера на другие серверы для соответствующего класса сервиса.

При анализе функциональной живучести систем различного назначения рассматриваются следующие характеристики системы: цель функционирования, множество задач, на решение которых система ориентирована, и множество ресурсов, используемых в процессе решения задач [1]. Определим характеристики системы управления - ИНДПУ.

Цель функционирования - предоставление ИС.

Множество задач (функций системы) - управление процессом предоставления ИС.

Множество ресурсов - множество функциональных элементов, используемых в процессе предоставления ИС.

При достижении цели функционирования системы управления для обеспечения живучести, применяют $s$ стратегию (survivability) [1]. При формировании $s$ стратегии следует определить множество состояний системы $S=\left\{s_{i}\right\}$, в каждом из которых осуществляется процесс управления предоставлением ИС $i$-го вида $\left(\mathrm{ИC}_{i}\right)(i=\overline{1, n}, n$ - число состояний системы (видов ИС))

В соответствии с [1] могут быть использованы следующие решения (механизмы) относительно цели функционирования:

1. Множество функций системы не может быть изменено, т.е. должны использоваться все функции $f_{i}$;

2. В любом состоянии системы должно выполняться некоторое подмножество функций $f^{*}$, которые реализуют цель функционирования системы;

3. В произвольном состоянии системы должна выполняться хотя бы одна функция из подмножества $f^{*}$.

Определим множество функций системы: $F=\left\{f_{1}, f_{2}, \ldots, f_{i}, \ldots, f_{n}\right\}$, где $f_{i}-$ функция управления предоставлением ИС $i$-го вида - ИС ${ }_{i}, n-$ количество видов функций системы (видов ИС).

Множество функциональных элементов $R=\left\{r_{1}\right.$, $\left.r_{1}, \ldots, r_{j}, \ldots, r_{m}\right\}$, где $r_{j}$ - функциональный элемент $j$-го вида, $m$ - количество видов функциональных элементов. Под $j$-м функциональным элементом будем понимать $j$-й вид ресурсов системы. Ресурсы $R$ используются в процессе выполнения функций $F$ системы. При этом для каждой $f_{i}$-й функции используется соответствующий набор ресурсов системы:

$$
\left\{r_{j}^{(i)}\right\} \subset R(j=\overline{1, m}) .
$$

Кроме того, задается множество функциональных связей $Y=\left\{y_{s t}\right\}$ между функциональными элементами $r_{j}(s, t=\overline{1, m}, s \neq t, j=\overline{1, m})$.

В соответствии с логикой и сложностью предоставления ИС объединим ИС в классы $K=\left\{K_{1}, K_{2}, \ldots, K_{q}, \ldots, K_{Q}\right\}$, где $q$ - класс ИС, $Q-$ количество классов. Для каждого класса $K_{q}$ используется соответствующий набор ресурсов $R^{q}=\left\{r_{j}^{q}\right\}$. Здесь $j=\overline{1, m}, \quad R^{q} \subset R, \quad q=\overline{1, Q}$. Задается также $Y^{q}=\left\{y_{f}^{q}\right\}-$ множество функциональных связей между элементами $r_{j}^{q}$, используемыми в процессе управления предоставлением ИС. На основе исходных множеств $R^{q}=\left\{r_{j}^{q}\right\}$ и $Y^{q}=\left\{\begin{array}{c}q \\ f\end{array}\right\}$ для каждого класса ИС формируется ориентированный граф без петель $G^{q}=\left(R^{q}, Y^{q}\right)$, где $R^{q}=\left\{r_{j}^{q}\right\}$ - вершины графа, $Y^{q}=\left\{y_{f}^{q}\right\}$ - функциональные связи между вершинами графа.

Процесс управления предоставлением ИС $q$-го класса (подмножество функций $f^{*}$ ) для ИНДПУ может быть представлен множеством маршрутов между пунктами А и Б - $\left\{\mu_{A Б}^{q}\right\}$ в графе $G^{q}=\left(R^{q}, Y^{q}\right)$ (здесь А пользователь, имеющий заявку на предоставление ИС, Б - сервер сервисов, исполняющий заявку). Каждый маршрут $\mu_{A D}^{q} \quad$ - последовательность вершин (множество используемых ресурсов) и ветвей (множество ветвей функциональных связей):

$$
\left\{\mu_{A B}^{q}\right\}=\left(\left\{r_{j}^{q}\right\},\left\{y_{s t}^{q}\right\} .\right.
$$

Отметим, что в выражении (2) множество используемых связей $\left\{y_{f}^{q}\right\}$ обозначено $\left\{y_{s t}^{q}\right\}$ для указания, между какими ресурсами ( $s$ и $t$ ) эта связь существует.

Представим z-й маршрут $\mu_{A Б}^{q}(z)$ множества $\left\{\mu_{A Б}^{q}\right\}$ между пунктами А и Б в виде:

$$
\mu_{A B}^{q}(z)=\left(r_{j A}^{q}(z), y_{A, 1}^{q}(z), r_{j 1}^{q}(z), y_{1,2}^{q}(z), \ldots, y_{(t-1), 5}^{q}, r_{j E}^{q}(z)\right) .
$$

Графически множество маршрутов $\left\{\mu_{A B}^{q}\right\}$ между пунктами А и Б (с номерами от 1 до $z$ ) представлено на рисунке 2 : 


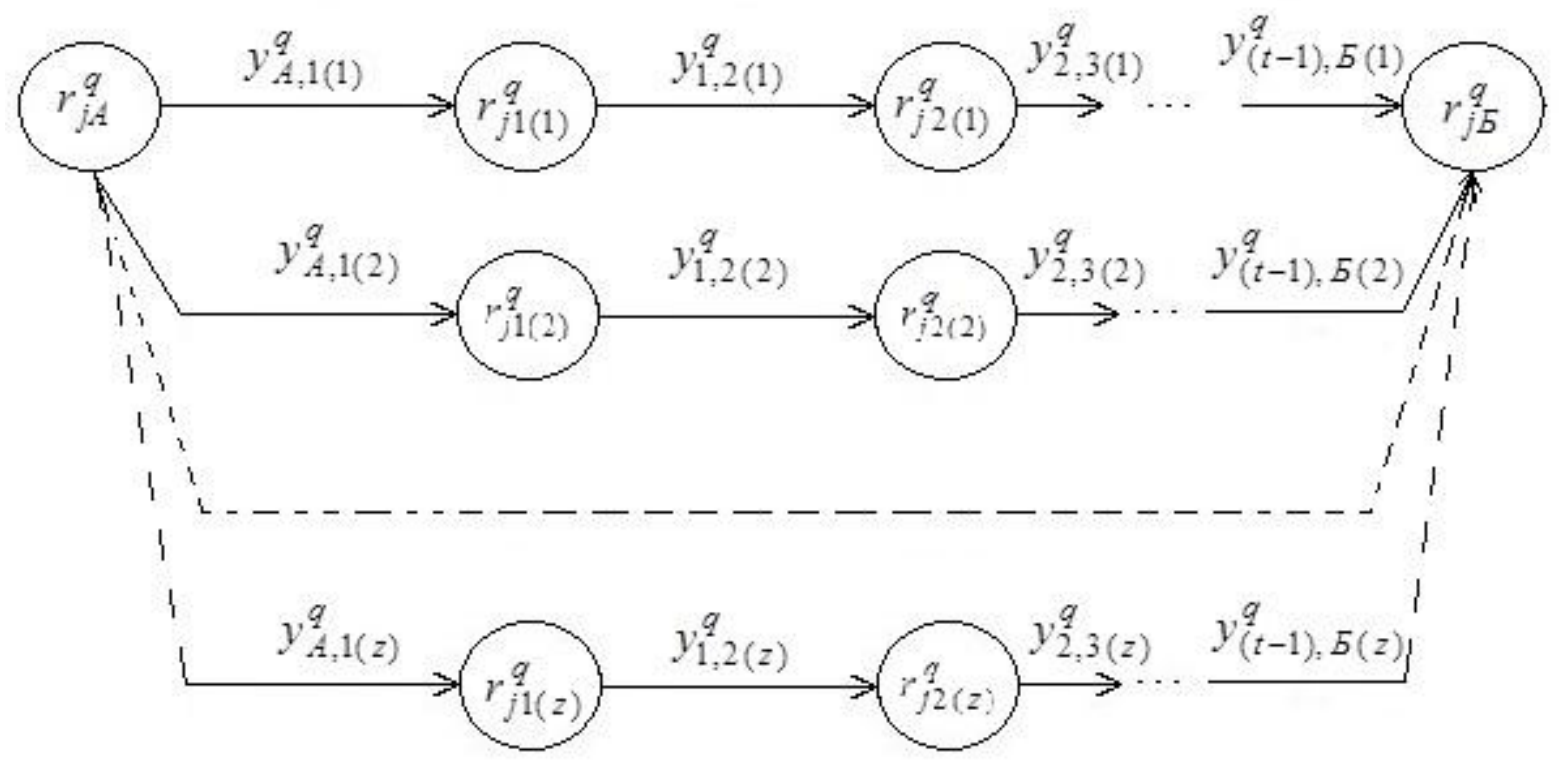

Рисунок 2 - Графическая интерпретация прочесса предоставления ИС q-го класса.

$$
3 \partial е c b\left(r_{j A}^{q}(z), r_{j 1}^{q}(z), \ldots, r_{j b}^{q}(z)\right) \subset R^{q},\left(y_{A, 1}^{q}(z), y_{1,2}^{q}(z), \ldots, y_{(t-1), B}^{q}\right) \subset Y^{q} .
$$

В соответствии с организацией интеллектуальной надстройки и с принципами предоставления ИС ресурсы $\mathrm{R}$ взаимодействуют на основе иерархического принципа (рисунок 1) [8]. Здесь множество $R$ составляют следующие ресурсы: сервер сервисов (Service Server), SG, MG, Softswitch, SCP (Service Control Point) -узел управления сервісами. Множество $y$ - связи взаимодействия между данными ресурсами.

Тогда выражение (2) может быть представлено следующим образом:

$$
\begin{aligned}
& \mu_{A B}^{q}(z)=\left(r_{j A\left(l_{A}\right)}^{q}(z), y_{A, 1\left(l_{A}+\delta_{A}\right)}^{q}(z),\right. \\
& r_{j 1\left(l_{1}\right)}^{q}(z), y_{1,2\left(l_{1}+\delta_{1}\right)}^{q}(z), r_{j 2\left(l_{2}\right)}^{q}(z), \\
& y_{2,3\left(l_{2}+\delta_{2}\right)}^{q}(z), \ldots, \\
& \left.y_{(t-1), 5\left(l_{(t-1)}^{q}+\delta_{(t-1)}\right)}^{q}(z), r_{j 5\left(l_{5}\right)}^{q}(z)\right),
\end{aligned}
$$

где $l_{A}, l_{1}, l_{2}, \ldots, l_{5}$ - характеристики принадлежности ресурсов $r_{j A}^{q}, r_{j 1}^{q}, r_{j 2}^{q}, \ldots, r_{j Б}^{q}$ соответствующему уровню иерархии, $l=\overline{1, L}$; $\left.\left.\left(l_{A}+\delta_{A}\right),\left(l_{1}+\delta_{1}\right),\left(l_{2}+\delta_{2}\right), \ldots,\left(l_{(} 1\right)+\delta_{(t} 1\right)\right)-$ характеристики принадлежности функциональных связей, исходящих из пунктов (ресурсов) $A, 1,2, \ldots$, используемых при предоставлении ИС по маршруту $\mu_{A Б}^{q}(z)$, соответствующему уровню иерархии.

Отметим, что при переходе от ресурса $r^{q}(z)$ к функциональной связи уровень иерархии может изменяться на $\delta_{s}(s=A, 1,2, \ldots)$, что демонстрирует рисунке 3 , на котором процесс предоставления ИС любого класса представлен в виде иерархической системы.

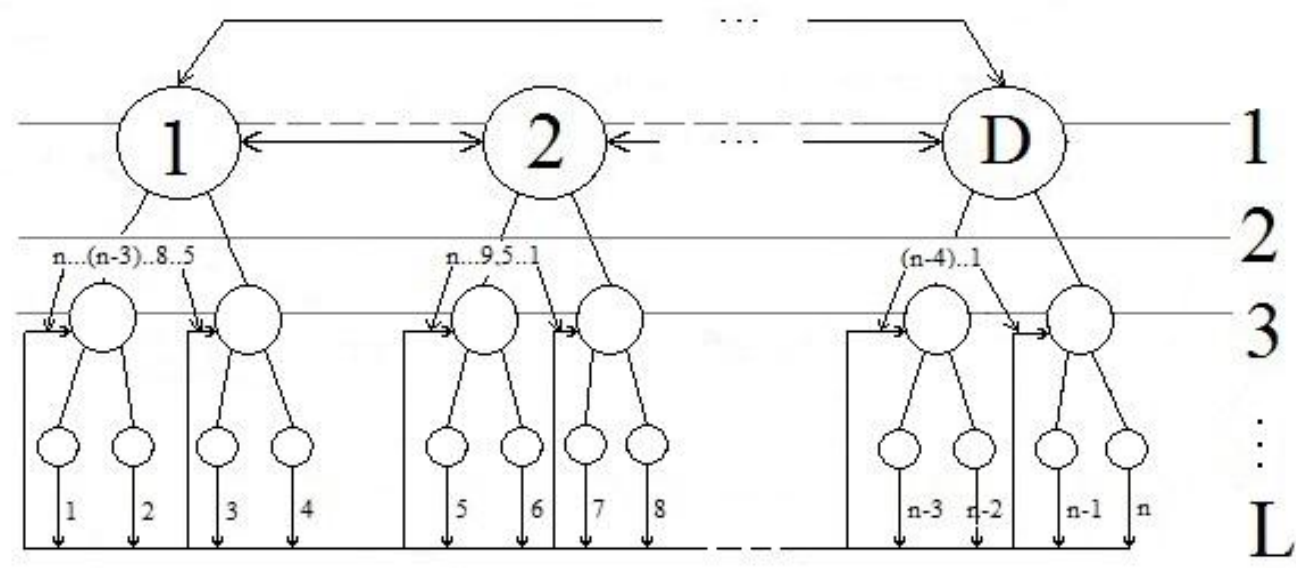

Рисунок 3 - Ярусы иерархической системы 
Как видно, на каждом ярусе с нечетной нумерацией расположены пункты (вершины графа, используемые ресурсы), с четной - ветви графа - функциональные связи между ресурсами системы.

Определим следующие количественные характеристики системы для каждого $d$-го территориального района $d=\overline{1, D}, D-$ количество территориальных районов. Будем считать все количественные характеристики районов идентичными и в обозначениях не применять идентификатор $d$ - номер района.

$N$ - общее количество пунктов (может совпадать со значением $m: N \geq m$ );

$B$ - общее количество ветвей;

$L$ - количество уровней иерархии;

$L_{r}-$ количество уровней пунктов;

$L_{y}-$ количество уровней функциональных связей;

$l$ - номер яруса (уровня), $l=\overline{1, L}$;

$l_{r}$ - номера уровней, на которых расположены пункты;

$l_{y}-$ номера уровней функциональных связей.

Будем считать, что все $D$ территориальных районов являются равноуязвимыми при внешних неблагоприятных воздействиях (ВНВ). Кроме того, будем считать также, что уровни иерархической системы для каждого $d$-го территориального района равноуязвимы.

В соответствии с рис. 3 номер верхнего уровня иерархии обозначим «1», следующего за ним уровня «2» и т.д. На каждом уровне $l(l=\overline{1, L})$ количество пунктов $r_{j}$ или ветвей (функциональных связей) $y_{s t}$ различно. Обозначим это количество $g_{l_{r}}$ и $g_{l_{y}}$ для уровней пунктов и ветвей, соответственно; $l_{r}=\overline{1, L_{r}}$, $l_{y}=\overline{1, L_{y}}$. Будем считать, что при ВНВ на уровень $l_{r}$ все пункты $q_{l}$ данного уровня равноуязвимы, а также что при ВНВ на уровень $l_{y}$ все функциональные связи данного уровня равноуязвимы.

Введем следующие события: $E_{H B}, E_{d}, E_{l}, E_{l_{r}}$, $E_{l_{y}}$.

1. $E_{H B}-$ возникновение ВНВ. Вероятность наступления этого события может быть сколь угодно малой, однако, всегда $P\left(E_{H B}\right)>0$.

2. $E_{d}$ - осуществление ВНВ в пределах $d$-го территориального района системы. Вероятность наступления этого события может быть сколь угодно малой, однако, всегда $P\left(E_{d}\right)>0$.Это событие может произойти только при наступлении события $E_{H B}$. Условная вероятность наступления события $E_{d}-$ $P\left\{E_{d} / E_{H B}\right\}$ - при условии осуществления события $E_{H B}$ определяется аксиомой вероятностей о совмещении событий $E_{d} \cap E_{H B}$ :

$$
P\left\{E_{d} \cap E_{H B}\right\}=P\left(E_{H B}\right) P\left(E_{d} / E_{H B}\right) .
$$

При этом $P\left(E_{d} / E_{H B}\right)=\frac{P\left(E_{d} / E_{H B}\right)}{P\left(E_{H B}\right)}$, отсюда и требование: $P\left(E_{H B}\right)>0$.

3. $E_{l}$ - осуществление ВНВ на $l$-й уровень $d$-го территориального района системы. Это событие может произойти только при наступлении события $E_{d}$.

Условная вероятность наступления события $E_{l}-$ $P\left\{E_{l} / E_{d}\right\}$ - при условии осуществления события $E_{d}$ определяется аксиомой вероятностей о совмещении событий $E_{l} \cap E_{d}$ :

$$
P\left\{E_{l} \cap E_{d}\right\}=P\left(E_{d}\right) P\left(E_{l} / E_{d}\right) .
$$

При этом, $P\left(E_{l} / E_{d}\right)=\frac{P\left(E_{l} / E_{d}\right)}{P\left(E_{d}\right)}$, отсюда и требование: $P\left(E_{d}\right)>0$.

4. $E_{l_{r}}-$ осуществление ВНВ на пункт $r_{j} l$-го уровня. Данное событие может произойти только в результате наступления события $E_{l}$.

Вероятность наступления события $E_{l_{r}}$ может быть определена как вероятность совмещения всех событий $E_{H B}, E_{d}, E_{l}, E_{l_{r}}:$

$$
\begin{aligned}
& P\left\{E_{H B} \cap E_{d} \cap E_{l} \cap E_{l_{r}}\right\}=P\left(E_{H B}\right) P\left(E_{d} \cap E_{H B}\right) \times \\
& \times P\left(E_{l} / E_{H B} \cap E_{d}\right) P\left(E_{l_{r}} / E_{H B} \cap E_{d} \cap E_{l}\right)
\end{aligned}
$$

5. $E_{l_{y}}-$ осуществление ВНВ на ветвь $y_{s t} l$-го уровня. Данное событие может произойти только в результате наступления события $E_{l}$.

Вероятность наступления события $E_{l_{y}}$ может быть определена как вероятность совмещения всех событий $E_{H B}, E_{d}, E_{l}, E_{l_{y}}:$

$$
\begin{aligned}
& P\left\{E_{H B} \cap E_{d} \cap E_{l} \cap E_{l_{y}}\right\}=P\left(E_{H B}\right) P\left(E_{d} \cap E_{H B}\right) \times \\
& \times P\left(E_{l} / E_{H B} \cap E_{d}\right) P\left(E_{l_{y}} / E_{H B} \cap E_{d} \cap E_{l}\right)
\end{aligned}
$$

Определив вероятности наступления событий $E_{l_{r}}$ и $E_{l_{y}}$ в соответствии с выражениями (4) и (5) и считая, что при наступлении события $E_{l_{r}}$ или $E_{l_{y}}$ происходит функциональный отказ элемента $r_{j}$ или элемента $y_{s t}$, можем определить вероятность отказа в предоставлении всех видов сервиса соответствующего $q$-го класса $P_{И C(q)}$, т.е. отказа в выполнении функций из подмножества $f^{*}$.

Как уже отмечалось, предоставление ИС $q$-го класса осуществляется с использованием маршрутов $\left\{\mu_{A D}^{q}\right\}$, таким образом, функциональный отказ всех маршрутов $\left\{\mu_{A Б}^{q}\right\}$ приводит к отказу в предоставлении ИС $q$-го класса, т.е. отказа в выполнении функций из подмноже- 
ства $f^{*}$ Поскольку функциональный отказ хотя бы одного элемента, составляющего маршрут (3), приводит к функциональному отказу маршрута, получим выражение для расчета вероятности функционального отказа маршрута на основе вероятности логически связанных событий, а именно, как вероятность осуществления хотя бы одного из $U$ независимых в совокупности событий $E_{1}, E_{2}, \ldots, E_{u}, \ldots, E_{U}$, где $E_{u}$ - событие, состоящее в функциональном отказе $u$-го элемента маршрута (3), $U$ - количество элементов маршрута:

$$
\begin{aligned}
& P\left(E_{1} \cup E_{2} \cup \ldots \cup E_{u} \cup \ldots \cup E_{U}\right)= \\
& =1-\left(1-P\left(E_{1}\right)\right)\left(1-P\left(E_{2}\right)\right) \ldots \\
& \left(1-P\left(E_{u}\right)\right) \ldots\left(1-P\left(E_{U}\right)\right)
\end{aligned}
$$

$P\left(E_{u}\right)$ - вероятность функционального отказа $u$-го элемента маршрута (3).

Обозначим событие, состоящее в функциональном отказе при ВНВ элементов маршрута (3), так:

1. $E\left(r_{j s\left(l_{s}\right)}^{q}(z)\right)$ - отказ ресурса $j$-го вида, используемого в процессе предоставления ИУ $q$-го класса по маршруту (3), включающему пункт $s$, расположенном на уровне иерархии $l_{s}$. Обозначение $《 l_{s}$ » используется в смысле указания принадлежности пункта $s$ маршрута (3) уровню $l(l=\overline{1, L})$.

2. $E\left(y_{j s\left(l_{s}+\delta_{s}\right)}^{q}(z)\right)-$ отказ функциональной связи, принадлежащей маршруту (3) и расположенной на уровне $\left(l_{s}+\delta_{s}\right)$, связанным с уровнем $l_{s}$.

Аналогично обозначим все другие события. Тогда выражение (6) может быть представлено в виде:

$$
\begin{aligned}
& P\left(E\left(r_{j A\left(l_{A}\right)}^{q}(z)\right) \cup E\left(y_{A, 1\left(l_{A}+\delta_{A}\right)}^{q}(z)\right) \cup E\left(r_{j 1\left(l_{1}\right)}^{q}(z)\right) \cup\right. \\
& \cup E\left(y_{1,2\left(l_{1}+\delta_{1}\right)}^{q}(z)\right) \cup E\left(r_{j 2\left(l_{2}\right)}^{q}(z)\right) \cup E\left(y_{2,3\left(l_{2}+\delta_{2}\right)}^{q}(z)\right) \cup \ldots \\
& \left.\ldots \cup E\left(y_{(t-1), 5\left(l_{t-1}+\delta_{t-1}\right)}^{q}(z)\right) \cup E\left(r_{j 5\left(l_{5}\right)}^{q}(z)\right)\right)= \\
& =1-\left(1-P\left(E\left(r_{j A\left(l_{A}\right)}^{q}(z)\right)\right)\right)\left(1-P\left(E\left(y_{A, 1\left(l_{A}+\delta_{A}\right)}^{q}(z)\right)\right)\right) \ldots \\
& \left.\ldots\left(1-P\left(E\left(y_{(t-1), 5\left(l_{t-1}+\delta_{t-1}\right)}^{q}(z)\right)\right)\right)\right)\left(1-P\left(E\left(r_{j 5\left(l_{5}\right)}^{q}(z)\right)\right)\right) .
\end{aligned}
$$

Здесь $\delta_{A}, \delta_{1}, \delta_{2}, \ldots$ - определяют изменение номера уровня $l$ при движении от пункта к ветви по маршруту $\mu_{A B}^{q}(z)$.

Для расчета вероятности функционального отказа маршрутов $\left\{\mu_{A Б}^{q}\right\}$ в результате ВНВ на любой элемент маршрута (3) следует определить значения всех вероятностей событий, входящих в выражение (7). Напомним, что количество элементов - пунктов и ветвей функциональных связей, находящихся на каждом $l$-м уровне, обозначены через $g_{l_{r}}$ и $g_{l_{y}}$, соответственно. При этом должны выполняться условия (8):

$$
\begin{gathered}
\sum_{\mathfrak{L}_{\gamma}=1}^{l_{\gamma}} n_{L_{\gamma}}=N ; \\
\sum_{\mathfrak{L}_{y=1}{ }_{y}}^{l_{L_{y}}}=B ; \\
L_{L_{\gamma}}+L_{y^{\prime}}=L .
\end{gathered}
$$

Исходя из гипотезы о равноуязвимости всех уровней системы (рисунок 3), рассчитаем условную вероятность событий, состоящих в ВНВ на уровень $l_{r}$ пунктов системы $P\left(E_{l_{r}}\right)(9)$ и уровень $l_{y}$ функциональных связей $P\left(E_{l_{y}}\right)(10)$ :

$$
\begin{aligned}
& P\left(E_{l_{r}}\right)=P\left(E_{H B} \cap E_{d} \cap E_{r}\right) \cdot \frac{L_{r}}{L_{r}+L_{y}} ; \\
& P\left(E_{l_{r}}\right)=P\left(E_{H B} \cap E_{d} \cap E_{l}\right) \cdot \frac{L_{y}}{L_{r}+L_{y}} ;
\end{aligned}
$$

Исходя из гипотезы о равноуязвимости всех элементов каждого уровня при ВНВ на данный уровень, получим выражения для расчета вероятностей поражения элементов уровней $l_{r}$ и $l_{y}-P_{l_{r}}^{q}$ и $P_{l_{y}}^{q}$, используемых в процессе предоставления ИС $q$-го класса с использованием маршрута $\mu_{A Б}^{q}(z)$.

Будем считать известными количество видов ресурсов $l_{r}\left(g_{l_{r}}^{q}\right)$ и функциональных связей $l_{y}\left(g_{l_{y}}^{q}\right)$, используемых для предоставления ИС $q$-го класса, находящихся на уровнях $l_{r}$ и $l_{y}$, соответственно.

С использованием введенных обозначений, получим:

$$
\begin{aligned}
& P_{l_{r}}^{q}=\frac{l_{r}\left(g_{l_{r}}^{q}\right)}{g_{l_{r}}} \cdot P\left(E_{H B} \cap E_{d} \cap E_{l} \cap E_{l_{r}}\right)= \\
& =\frac{l_{r}\left(g_{l_{r}}^{q}\right)}{g_{l_{r}}} \cdot \frac{L_{r}}{L_{r}+L_{y}} \cdot P\left(E_{H B} \cap E_{d} \cap E_{l}\right), \\
& P_{l_{y}}^{q}=\frac{l_{y}\left(g_{l_{y}}^{q}\right)}{g_{l_{y}}} \cdot P\left(E_{H B} \cap E_{d} \cap E_{l} \cap E_{l y}\right)= \\
& =\frac{l_{y}\left(g_{l_{y}}^{q}\right)}{g_{l_{y}}} \cdot \frac{L_{y}}{L_{r}+L_{y}} \cdot P\left(E_{H B} \cap E_{d} \cap E_{l}\right) .
\end{aligned}
$$

Таким образом, выражения (8),...,(12) дают возможность рассчитать вероятность функционального отказа маршрута $\mu_{A B}^{q}(z)$

Обозначив указанную вероятность через $P(z)$ и общее количество используемых маршрутов через $Z$, получим выражение для расчета показателя функциональной живучести системы $P_{\text {Исж(q) }}-$ способности выполнять функции из подмножества $f^{*}$ : $P_{\text {ИСж }}(q)=1-\prod_{z=1}^{Z} P(z)$.

Вероятность отказа в предоставлении $i$-го вида сервиса $\boldsymbol{q}$-го класса ИС , т.е. отказа в выполнении хотя бы одной функции из подмножества $f^{*}-P_{\text {ИCi(q) }}$, может быть получена исходя из следующего.

Вероятность осуществления хотя бы одного из двух событий $E_{p}-$ функционального отказа ресурсов 
уровня $l_{r}(11)$ или $E_{\phi}$ - отказа функциональных связей уровня $l_{y}$ (12), используемых в маршрутах $\left\{\mu_{A Б}^{q}\right\}$ при предоставлении всех видов сервиса соответствующего $q$-го класса $-P_{И C(q)}$, может быть рассчитана как вероятность осуществления хотя бы одного из двух событий $E_{p}$ и $E_{2}$ :

$$
P\left\{E_{p} \cup E_{\phi}\right\}=P\left\{E_{p}\right\}+P\left\{E_{\phi}\right\}-P\left\{E_{p} \cap E_{\phi}\right\}
$$

В результате вероятность отказа в предоставлении $i$ го вида сервиса $q$-го класса ИС $_{i}-$ выполнении хотя бы одной функции из подмножества $f^{*}-P_{\text {ИСi(q) }}$ определяется в соответствии с выражением (14):

$$
P_{И C_{i}(q)}=1-\left(P\left\{E_{p}\right\}+P\left\{E_{\phi}\right\}-P\left\{E_{p} \cap E_{\phi}\right\}\right) .
$$

Будем считать известными количество видов ресурсов $l_{r}\left(g_{l_{r i}}^{q}\right)$ и функциональных связей $l_{y}\left(g_{l_{y i}}^{q}\right)$, используемых для предоставления ИС ${ }_{i} q$-го класса, находящихся на уровнях $l_{r}$ и $l_{y}$, соответственно.

С использованием введенных обозначений получим способ расчета вероятностей поражения элементов уровней $l_{r}$ и $l_{y},-P_{l_{r i}}^{q}(15)$ и $P_{l_{y i}}^{q}(16)$, используемых в процессе предоставления ИС $q$-го класса:

$$
\begin{aligned}
P_{l_{r i}}^{q} & =\frac{l_{r}\left(g_{l_{r i}}^{q}\right)}{l_{r}\left(g_{l_{r}}^{q}\right)} \cdot \frac{l_{r}\left(g_{l_{r}}^{q}\right)}{g_{l_{r}}} \cdot \frac{L_{r}}{L_{r}+L_{y}} \cdot P\left(E_{H B}\right), \\
P_{l_{y}}^{q} & =\frac{l_{y}\left(g_{l_{y i}}^{q}\right)}{l_{y}\left(g_{l_{y}}^{q}\right)} \cdot \frac{l_{y}\left(g_{l_{y}}^{q}\right)}{g_{l y}} \cdot \frac{L_{y}}{L_{r}+L_{y}} \cdot P\left(E_{H B}\right) .
\end{aligned}
$$

Здесь $i=\overline{1, n}, l_{r}=\overline{1, L_{r}}, l_{y}=\overline{1, L_{y}}, q=\overline{1, Q}$.

Рассчитав в соответствии (15) и (16) значения $P_{l_{r i}}^{q}$ и $P_{l_{y i}}^{q}$ и подставив полученные результаты в выражение (7), получим искомый показатель вероятности функционального отказа хотя бы одного элемента, составляющего маршрут предоставления ИС ${ }_{i}$, т.е. получим вероятность нарушения функциональной живучести системы в отношении предоставления $i$-го сервиса $q$-го класса.

Вероятность сохранения функциональной живучести системы $P_{И C_{i} ж(q)}$ - выполнения хотя бы одной функции из множества $f^{*}$ (предоставления хотя бы одного вида ИС $i q$-го класса) - получим как вероятность противоположного события (17):

$$
\begin{aligned}
& P_{U C_{i} ж(q)}=1-\left(P \left(E\left(r_{j A\left(l_{A}\right)}^{q}\right) \cup E\left(y_{A, 1\left(l_{A}+\delta_{A}\right)}^{q}\right) \cup E\left(r_{j 1\left(l_{1}\right)}^{q}\right) \cup\right.\right. \\
& \cup E\left(y_{1,2\left(l_{1}+\delta_{1}\right)}^{q}\right) \cup E\left(r_{j 2\left(l_{2}\right)}^{q}\right) \cup E\left(y_{2,3\left(l_{2}+\delta_{2}\right)}^{q}\right) \cup \ldots \\
& \left.\left.\ldots \cup E\left(y_{(t-1), E\left(l_{t-1}+\delta_{t-1}\right)}^{q}\right) \cup E\left(r_{j E\left(l_{5}\right)}^{q}\right)\right)\right) .
\end{aligned}
$$

\section{3 Выводы}

Таким образом, получены выражения для оценки функциональной живучести системы предоставления интеллектуального сервиса - выполнения некоторого подмножества функций $f^{*}$, которые реализуют цель функционирования системы (предоставления ИС), а также выполнения хотя бы одной функции из подмножества $f^{*}$ (предоставления ИС ${ }_{i} q$-го класса).

Полученные выражения могут быть использованы при проектировании системы управления интеллектуальным сервисом, при выборе принципа управления, при решении задач повышения функциональной живучести системы управления интеллектуальным сервиcom.

\section{Литература}

1. Додонов А.Г. Живучесть информационных систем [Текст] / А.Г. Додонов, Д.В. Ландэ. - К.: Наук. думка, 2011. $-256 \mathrm{c}$.

2. Стекольников Ю.И. Живучесть систем [Текст] / Ю.И. Стекольников - СПб.: Политехника, 2002. - 155c.

3. Князева Н.А. Повышение структурной живучести телекоммуникационной сети [Текст] / Н.А. Князева // International Journal "Information Models and Analyses". — 2013. - №. 2. - С. 275-284.

4. Князева Н.А. Метод обеспечения структурной живучести телекоммуникационной сети [Текст] / Н.А. Князева // International Journal "Information Technologies \& Knowledge”. — 2014. - №. 8. - C. 152-166.

5. Kniazieva N. Complex Quality Criterion of Control of the Intellectual Services Provision in NGN [Text] / N Kniazieva, L. Zimenko, T. Kunup // Science and Education a New Dimention: Natural and Technical Science. -2016. IV(10), Issue 91. P. 79-85.

6. Князева Н.А. Метод обеспечения структурной живучести интеллектуальной надстройки [Текст] / Н.А. Князева, Л.Н. Зименко// Інформаційно-керуючі системи на залізничному транспорті. - №6 . - 2016. - С. 23-29.

7. Шестопалов C. В. Architecture of NGN with centralized and decentralizing control system [Електронний ресурс] / С. В. Шестопалов // 10-та Ювілейна міжнародна науково-технічна конференція "Сучасні проблеми радіоелектроніки, телекомунікацій та комп'ютерної інженерії" TCSET'2010. - Львів. - 1 електрон. опт. диск (CD-ROM); 12 см. - Систем. вимоги: Pentium; 32 Mb RAM; CD-ROM; Windows 95/98/2000/NT/XP; MS Word 97-2003.

Отримана в редакції 23.02.2017, прийнята до друку 25.04.2017 


\title{
Method for Determining the Functional Survivability in Case of Decentralized Control of Intelligent Service
}

\section{N. A. Kniazieva}

Odessa National Academy of Food Technologies, 112 Kanatna str., Odesa, 65039, Ukraine

\begin{abstract}
In this paper the method for determining the functional survivability of intelligent superstructure, that performs decentralized control of the process of providing intelligent services is proposed and also are obtained formulae for estimation functional survivability for providing intelligent services the performance a subset of functions that realize the purpose of the functioning of the system (the provision of intelligent services of a certain class), and also performance of at least of one of the functions from a subset (providing at least one kind of certain class intelligent service). The received formulae can be objectively used at the design of the control system over the intelligent service, at the choice of a control principle, at the decision of the problems of control system functional survivability increasing over intelligent service.
\end{abstract}

Keywords: Functional Survivability; Intelligent Superstructure; Decentralized Control; Intelligent Service.

\section{References}

1. Dodonov, A.G., Lande, D.V. (2011) Zhivuchest informatsionnyih system. Kyiv, Naukova dumka, 256 p.

2. Stekolnikov, Yu.I. (2002) Zhivuchest system. SPb., Politehnika, 155 p.

3. Kniazieva, N.A. (2013) Povyishenie strukturnoy zhivuchesti telekommunikatsionnoy seti. Information Models and Analyses, No.2, 275-284.

4. Kniazieva, N.A. Metod obespecheniya strukturnoy zhivuchesti telekommunikatsionnoy seti. Information Technologies \& Knowledge, No. 8, 152-166.

5. Kniazieva, N., Zimenko, L., Kunup, T. (2016) Complex Quality Criterion of Control of the Intellectual Services Provision in NGN. Science and Education a New
Dimention: Natural and Technical Science, IV(10), iss. 91, 79-85.

6. Kniazieva, N. A., Zimenko, L.N. (2016) Metod obespecheniya strukturnoy zhivuchesti intellektualnoy nadstroyki. Informatsiyno-keruyuchi sistemi na zaliznichnomu transporti, No.6, 23-29.

7. Shestopalov S. V. (2010) Architecture of NGN with centralized and decentralizing control system [Elektronic source] 10-ta Yuvileyna mizhnarodna naukovo-tehnichna konferentsiya "Suchasni problemy radioelektroniky, telekomunikatsii ta kompyuternoi inzhenerii" TCSET'2010. LvIv. (CD-ROM).

Received 23 February 2017 Approved 25 April 2017 Available in Internet 29 May 2017 